\title{
In vitro antifungal susceptibility testing of Candida isolates from women with recurrent vulvovaginal candidiasis
}

\author{
Stavroula Antonopoulou, ${ }^{1} \mathrm{MD} \mathrm{PhD}$, Drosos Karageorgopoulos, ${ }^{2} \mathrm{MD} \mathrm{PhD} \mathrm{MPH}$, \\ Joseph Meletiadis, ${ }^{3} \mathrm{PhD}$, Flavia De Bernardis, ${ }^{4} \mathrm{PhD}$
}

\author{
${ }^{1}$ Dept of Biology, University of Athens, Greece, ${ }^{2}$ Dept of Medicine, Attikon University \\ General Hospital, Athens, Greece, ${ }^{3}$ Clinical Microbiology Laboratory, Attikon University \\ General Hospital, Athens, Greece, ${ }^{4}$ Istituto Superiore di Sanita , Rome, Italy
}

\begin{abstract}
Objectives
Vulvovaginal canididiasis (VVC) is a common infection among premenstrual women. Around $10-20 \%$ of women will have recurrent VVC (RVVC) (four or more episodes per year). This is often due to non-albicans Candida spp., which have reduced susceptibility to antifungal agents. Due to the lack of clinical breakpoints for topical antifungals, epidemiological cut-off values may be used to detect non-wild type isolates. We aimed to determine the ECOFFs of RVVC isolates to different antifungal agents and identify the best indicator drugs to be used for routine screening for resistant isolates.
\end{abstract}

\section{Methods:}

More than 500 Candida spp. isolates have been collected over the past 10 years from women with RVVC at MycoLab outpatient Microbiology Laboratory, Athens, Greece and were stored at $-70^{\circ} \mathrm{C}$. They were revived by subculturing at $35^{\circ} \mathrm{C}$ on Chromagar plates. Species identification was performed with Auxacolor (Biorad) and additional techniques, as required. Inocula were prepared and adjusted to $0.5 \mathrm{McF}$ arland standard. The following antifungal agents were used for susceptibilty testing according to the EUCAST E.Def. 7.3 protocol: clotrimazole, econazole, fluconazole, itraconazole, ketoconazole, miconazole, and boric acid. Two-fold serial dilutions of the drugs were prepared in 96well microplates. The final drug concentrations ranged from 0.016 to $16 \mathrm{mg} / \mathrm{l}$. The final inoculum was in the $1-5 \times 10^{5} \mathrm{CFU} / \mathrm{ml}$ range.

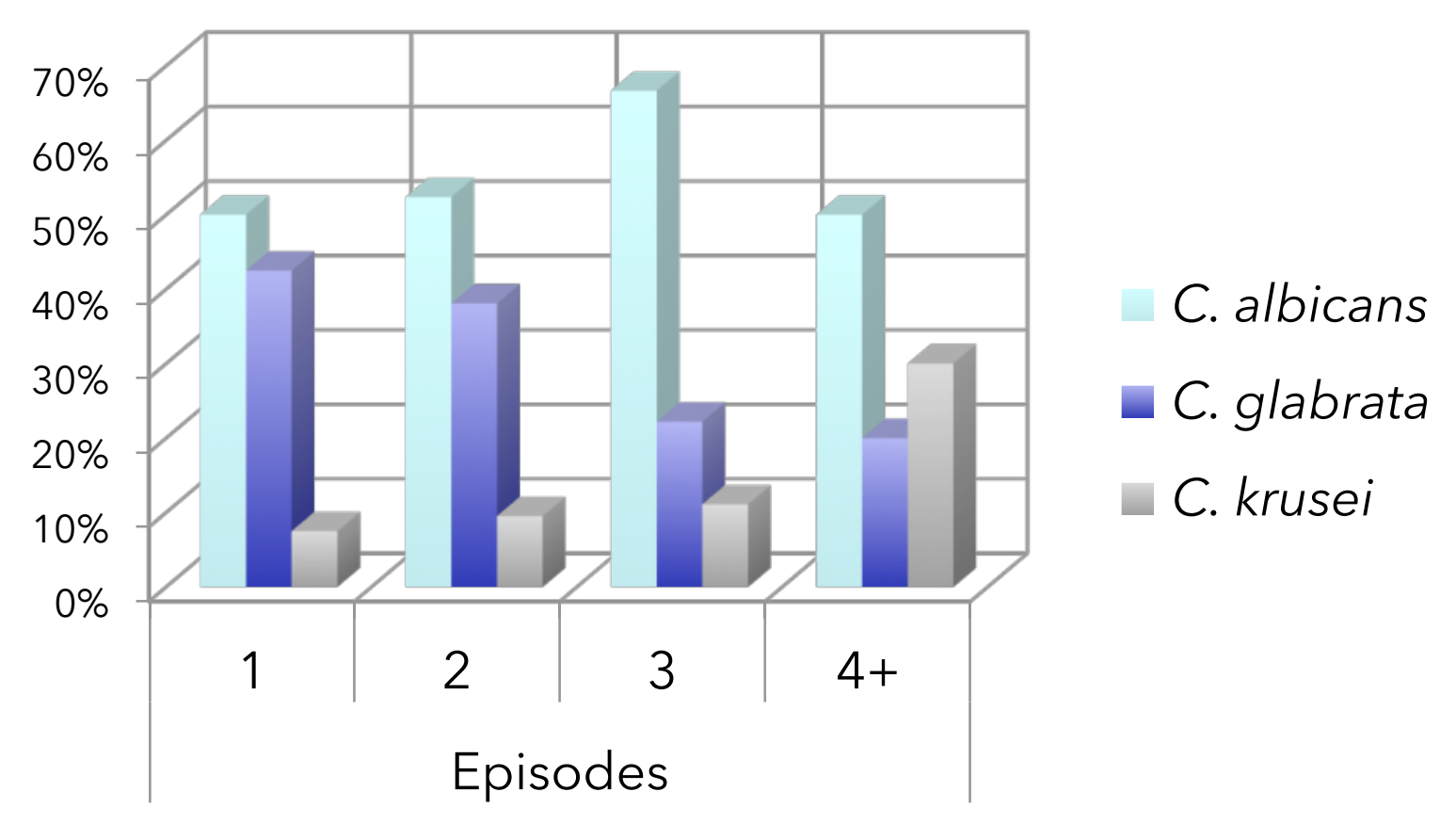

Figure. Distribution of isolates from women with episodes of vulvovaginal candidiasis, according to the number of episodes
Microplates were incubated at $35^{\circ} \mathrm{C}$ for $24 \mathrm{~h}$ and the optical density OD of each well was measured at 530nm. The MIC was determine as the lowest drug concentration producing $>50 \%$ growth inhibition. The median $\mathrm{MICs}, \mathrm{MIC}_{90}$ and the MIC range was determined for each drug and Candida spp combination. The epidemiological cut-off values (ECOFFs) were determined with the ECOFFinder calculator. The \% of isolates with an MIChigher than the ECOFF reflecting non wild-type isolates was determined. The differences in the MICs among different drugs were analyzed with AVONA followed by multiple comparison test after $\log _{2}$ transformation. In order to assess cross-resistance, the $\log _{2}$ MICs of each drug was correlated with the $\log _{2}$ MICs of the other drugs using Pearson analysis.

\section{Results:}

Our preliminary results show a high percentage of Candida glabrata and Candida krusei among women with recurrent episodes of Candida vulvovaginitis (Figure). The final results of this study will help to determine the epidemiology and in vitro susceptibility of Candida isolates from women with RVVC. The in vitro susceptibility to different drugs used to treat RVVC will be compared. Furthermore, epidemiological cutoff values will be determined for each Candida species. The ECOFF values will help to detect non-wild type isolates with reduced susceptibility and possible poor clinical outcome. Drugs indicators of in vitro susceptibility will be identified in order to facilitate clinical labs to screen fast with minimal requirements Candida RVVC isolates. Finally, the findings of this study will be communicated to the EUCAST subcommittee for antifungal susceptibility testing in order to formulate guidelines for isolates involved in RVVC.

\section{Conclusion:}

RVVC is often caused by non-albicans Candida isolates, that have reduced in vitro susceptibility to antifungal drugs. Previous exposure to topical or oral antifungals in women with RVVC may lead to accumulation of resistance mechanisms. We hope that our study will clarify the need for antifungal susceptibility testing in women with RVVC for therapeutic and epidemiological purposes. 